\title{
Individual Subjective Initiative Merge Model Based on Cellular Automaton
}

\author{
Yin-Jie Xu, ${ }^{1}$ Yu-Guang Chen, ${ }^{1}$ Chao Yang, ${ }^{2}$ and Yi-Chao Pu ${ }^{2}$ \\ ${ }^{1}$ Pohl Institute of Solid State Physics, Tongji University, Shanghai 200092, China \\ ${ }^{2}$ Key Laboratory of Road and Traffic Engineering, Tongji University, Shanghai 201804, China \\ Correspondence should be addressed to Chao Yang; tongjiyc@163.com
}

Received 2 August 2012; Revised 2 January 2013; Accepted 3 January 2013

Academic Editor: Wuhong Wang

Copyright (C) 2013 Yin-Jie Xu et al. This is an open access article distributed under the Creative Commons Attribution License, which permits unrestricted use, distribution, and reproduction in any medium, provided the original work is properly cited.

The merge control models proposed for work zones are classified into two types (Hard Control Merge (HCM) model and Soft Control Merge (SCM) model) according to their own control intensity and are compared with a new model, called Individual Subjective Initiative Merge (ISIM) model, which is based on the linear lane-changing probability strategy in the merging area. The attention of this paper is paid to the positive impact of the individual subjective initiative for the whole traffic system. Three models (ISIM, HCM, and SCM) are established and compared with each other by two order parameters, that is, system output and average vehicle travel time. Finally, numerical results show that both ISIM and SCM perform better than HCM. Compared with SCM, the output of ISIM is 20 vehicles per hour higher under the symmetric input condition and is more stable under the asymmetric input condition. Meanwhile, the average travel time of ISIM is 2000 time steps less under the oversaturated input condition.

\section{Introduction}

Over the past several decades, many methods and rules [1-9] have been proposed for the work zone system which consists of a double-lane (DL) model and a single-lane (SL) model. Among them, many have been implemented or tested in practice and have been effective on the real traffic system. They are named after the characteristics of the model, the strategy, the authors and so on, but when we focus on the control intensity, we can briefly classify them into two types: (1) Hard Control Merge (HCM) model (whose control intensity is strong, such as lane-based signal system [10]); (2) Soft Control Merge (SCM) model (whose control intensity is mild, such as dynamic late merge system and lane-based dynamic merge system [11]). But both models ignore the impact of the individual subjective initiative for the whole system and it is the point that we focus on in this paper.

In next sections, three models with different lane-changing rules and a vehicle input method are introduced. Simulation results and analyses are presented after that. Conclusions and further studies are given in the last section.

\section{Model}

We introduce Cellular Automaton (CA) $[12,13]$ which is a discrete model and present three models based on CA. The CA model consists of a regular grid of cells with a finite number of states. For each cell, a set of cells called its neighborhood is defined relative to it. The selection of the initial state is determined by the model. A new generation is created according to some fixed rules which determine the new state of each cell with the consideration of the current states of the cell and its neighborhood. Typically, the rule to update the state of cells is the same for each cell and does not change over time. Above all, it is applied to the whole grid simultaneously.

2.1. Basic Updating Rules. The basic updating rules are introduced according to NS model [14], and the updating rule for the $n$th vehicle at $(t+1)$ th time step is as follows.

(1) Accelerating at the $(t+1 / 4)$ th time step

$$
V_{n, t+1}=V_{n, t}+1 \text {. }
$$


(2) Safety breaking at the $(t+2 / 4)$ th time step

$$
V_{n, t+1}=\min \left(V_{n, t}, d_{n, t}\right) \text {. }
$$

(3) Random decelerating at the $(t+3 / 4)$ th time step

$$
\begin{gathered}
V_{n, t+1}=\max \left(V_{n, t+1}-1,0\right) \quad \text { with probability } P_{d} \in[0,1], \\
V_{n, t+1}=V_{n, t+1} \quad \text { with probability } 1-P_{d} .
\end{gathered}
$$

(4) Moving at the $(t+1)$ th time step

$$
X_{n, t+1}=X_{n, t}+V_{n, t+1}
$$

where $V_{n, t}$ is the velocity of the $n$th vehicle at $t$ th time step, $X_{n, t}$ is the position of the $n$th vehicle at $t$ th time step, $d_{n, t}$ is the forward headway of the $n$th vehicle at $t$ th time step, and $P_{d}$ is the decelerating probability.

2.2. Basic Model. The basic model is presented in Figure 1. The work zone system is composed of a 100-lattice long DL model and a 100-lattice long SL model (one lattice is equal to 7.5 meters). We name the upper lane as Lane 1 and the other as Lane 2. We divide DL model into two parts: Part 1 is 94-lattice long and Part 2 is 6-lattice long. Part 1 is the core area of the model, and the basic model converts into different merge models when we introduce different lanechanging rules into Part 1 . We use different kinds of lanechanging probability $\left(P_{l} \in[0,1]\right)$ to simulate different lanechanging rules. The higher the lane-changing probability is, the more probably the vehicle changes its lane. Part 2 is the coercive lane-changing area. In this part, vehicles on Lane 1 have to change lane before the merge point and vehicles on Lane 2 are not allowed to change lane. Thus, in Part 2, the lane-changing probability of the vehicle on Lane 1 is always 1 , and the probability of the vehicle on Lane 2 is 0 .

2.3. Merge Models. We introduce three different kinds of lane-changing rules into the basic model and then get three models: (1) ISIM, (2) HCM, and (3) SCM.

(1) ISIM. The lane-changing probability of ISIM is shown in Figure 2 . Then we set the probability of the vehicle on Lane 1 changing to Lane 2 as follows:

$$
\begin{gathered}
P_{l, 1 \rightarrow 2}=0.5+0.5 \times \frac{L}{94} \quad L \leq 94, \\
P_{l, 1 \rightarrow 2}=1 \quad L>94,
\end{gathered}
$$

and the probability of the vehicle on Lane 2 changing to Lane 1 is

$$
\begin{gathered}
P_{l, 2 \rightarrow 1}=0.5-0.5 \times \frac{L}{94} \quad L \leq 94, \\
P_{l, 2 \rightarrow 1}=0 \quad L>94 .
\end{gathered}
$$

With the linear lane-changing probability, we try to perform a more realistic simulation and find out the impact of the individual subjective initiative on the whole system.

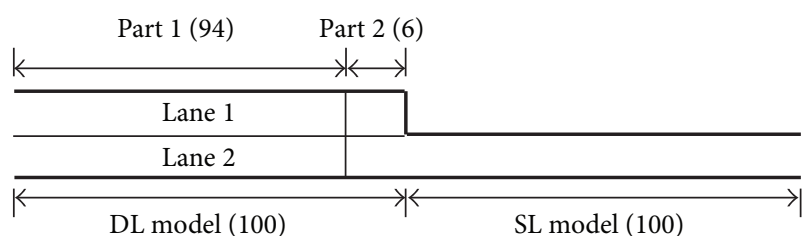

Figure 1: The basic work zone system.

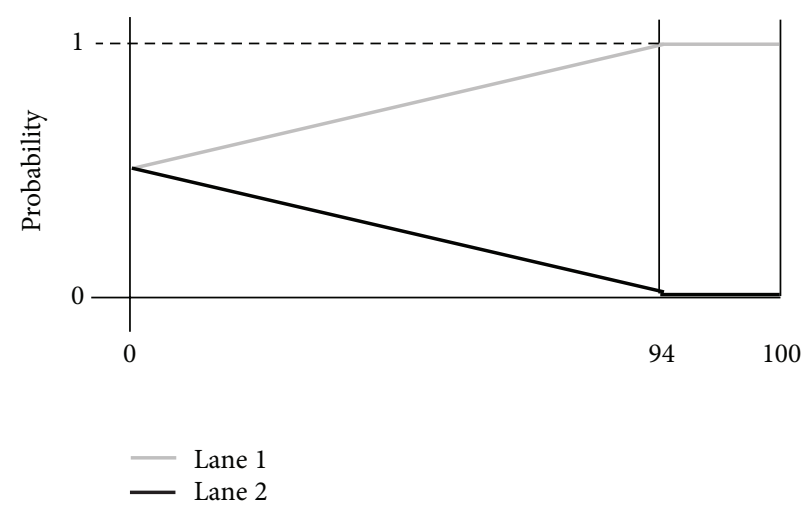

FIGURE 2: Lane-changing probability of ISIM.

(2) HCM. The lane-changing probability of HCM is presented in Figure 3. The two lanes are separated in Part 1. According to this, the lane change is not allowed and the vehicle will stay on the lane where it is injected at the beginning. We put a signal between the 94th lattice and the 95th lattice, and only one lane is allowed to drive in each interval according to the signal (the interval between two adjacent signal changes is set as 30 time steps). We can use this model to simulate lane-based signal system. 2 is

The probability of the vehicle on Lane 1 changing to Lane

$$
\begin{array}{ll}
P_{l, 1 \rightarrow 2}=0 & L \leq 94, \\
P_{l, 1 \rightarrow 2}=1 & L>94,
\end{array}
$$

and the probability of the vehicle on Lane 2 changing to Lane 1 is

$$
P_{l, 2 \rightarrow 1}=0 \text {. }
$$

(3) SCM. The lane-changing probability of SCM is illustrated in Figure 4. The probability of the vehicle on Lane 1 changing to Lane 2 is

$$
\begin{gathered}
P_{l, 1 \rightarrow 2}=0.5+0.5 \times \frac{L}{94} \quad L \leq 94, \\
P_{l, 1 \rightarrow 2}=1 \quad L>94,
\end{gathered}
$$

and the probability of the vehicle on Lane 2 changing to Lane 1 is

$$
P_{l, 2 \rightarrow 1}=0 \text {. }
$$

The difference between ISIM and SCM is the lane-changing probability of the vehicle on Lane 2 . When the traffic 


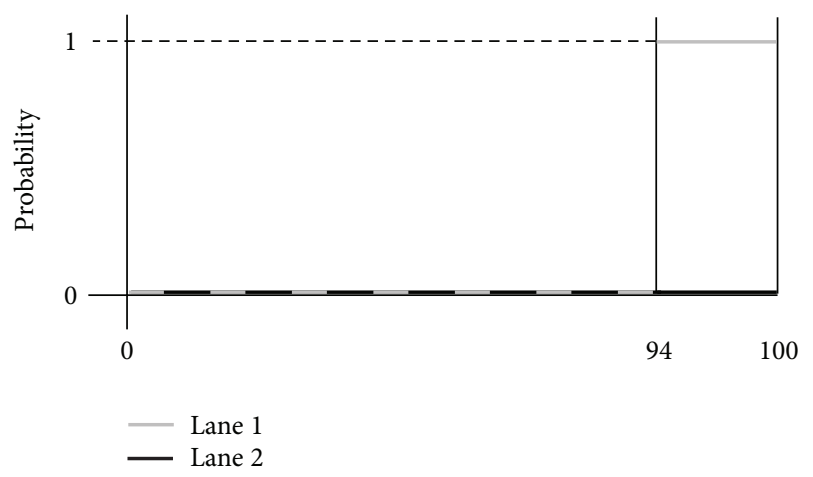

FIGURE 3: Lane-changing probability of HCM.

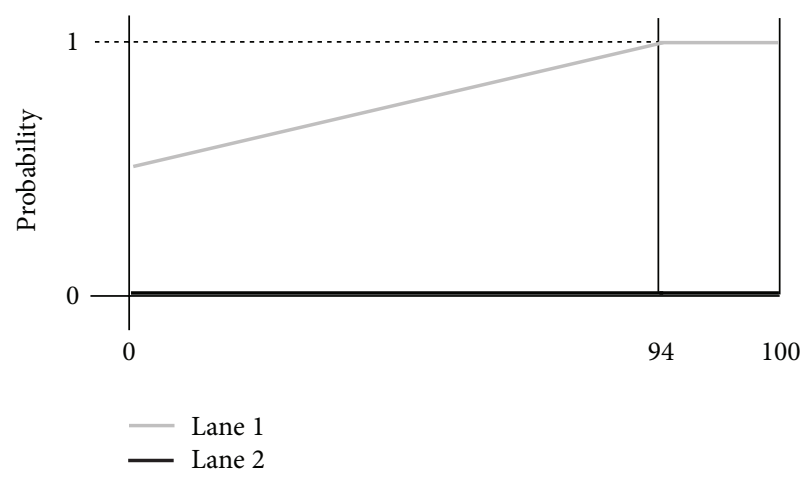

FIGURE 4: Lane-changing probability of SCM.

demand is light, vehicles on Lane 1 can easily change lane at the upstream of the DL model (like the static early merge in [11]). When the traffic demand is high and the model is congested, they may not easily change to Lane 2 at the upstream of the DL model and have to change lane near the end of the DL model (like the static late merge in [11]). Thus, with the increasing of the traffic demand, the real merge point will move backwards constantly. Meanwhile, the static early merge will convert into the static late merge gradually. According to this, we can use it to simulate the lane-based dynamic merge in some degree but we do not set a definite control threshold.

2.4. Vehicle Input Methods. We introduce the probability distribution function here to obtain the quantity of the arriving vehicle per time step. According to the traffic flow theory, the Poisson distribution function is adaptive under the light traffic condition and the binomial distribution function is chosen under the congested condition [15]. Here we set the threshold as 0.25 vehicles per time step (equal to 900 vehicles per hour (vph)).

The Poisson distribution function is

$$
P(x)=\frac{(\lambda t)^{x} e^{-\lambda t}}{x !},
$$

and the binomial distribution function is

$$
P(x)=C_{n}^{x}\left(\frac{\lambda t}{n}\right)^{x}\left(1-\frac{\lambda t}{n}\right)^{n-x}
$$

where $P(x)$ is the probability that $x$ vehicles arrive within $t$ time steps, $\lambda t$ is the quantity of the arriving vehicle within $t$ time steps (here $\lambda=0.25$ ), and $n$ is the maximum quantity of the arriving vehicle per time step.

After getting the quantity of arriving vehicles, we do not input them into the model directly. Instead, we put them into a stack and set the serial number and arriving time for them. Then we input them into the model in turn (according to the First-In-First-Out principle) with the consideration of the real-time conditions of the lane and the stack.

\section{Simulation Results and Analysis}

We performed simulations based on the three models described above with $v_{\max }=4$ lattice/time step. Then the results, such as the output and the travel time, are collected under different input conditions. All data are collected during the 3600 time steps from the 14401st time step to the 18000th time step.

3.1. Theoretical Output of Work Zone System. We simulate two other basic models for comparison: a 200-lattice long SL model and a 200-lattice long DL model. Briefly, the work zone model is composed of a SL model and a DL model, so the simulation of SL and DL model helps us to get the theoretical limitations of the output of the work zone system in Figure 5(a). For a better discussion, we make the fitting line of the output of SL and DL model in Figure 5(b). From it, we notice that Output max,DL $(=2600 \mathrm{vph})$ is larger than Output ${ }_{\max , \mathrm{SL}}(=$ $1160 \mathrm{vph}) \times 2$. A well-performing DL model can reduce the negative impact of the following vehicle [16]. According to this, it is the logical result of taking full advantage of DL model with safety lane-changing rules. The output of work zone system will be suppressed by the SL model, so the theoretical Output ${ }_{\max }$ of the work zone system is similar to 1160 vph. Compared with a SL model, a DL model can support a higher single-lane input value with the reasonable merge rules, so the theoretical value of work zone may be a little more than $1160 \mathrm{vph}$. We cannot get the exact number, so we use the result of the SL model as the theoretical value of the work zone system.

3.2. Output under Symmetric Input Conditions. We simulate the three models individually, and the results are illustrated in Figure 6(a). In Figure 6(b), we compare the results of three models with the theoretical value. The Output ${ }_{\text {max,ISIM }}$ is the largest, and both the Output max,ISIM $_{\text {and the Output }}$ max,SCM are better than the theoretical value. The good performance of ISIM and SCM is due to the individual subjective initiative, and vehicles in these two models can change lane with safety lane-changing rules. This behavior takes full advantage of DL model and led to better output results. As illustrated in Figure 6, it seems that ISIM result is only slightly better than that of SCM. However, we will find that the advantage of ISIM is more obvious compared with SCM when we observe the travel time presented in the following section. Compared with the others, the result of HCM is unsatisfactory and it is more inefficient not only than the other two models but 


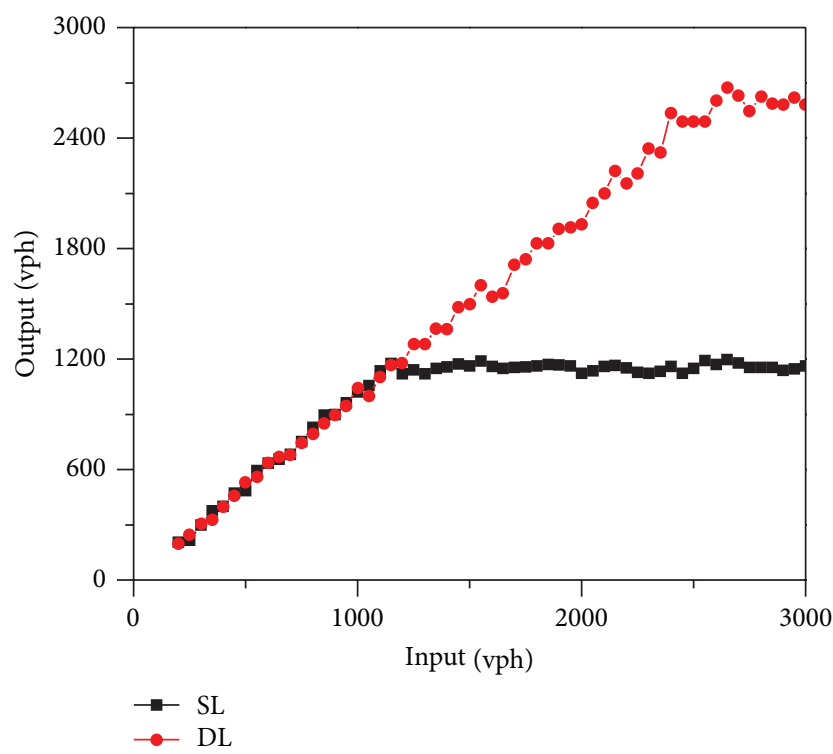

(a)

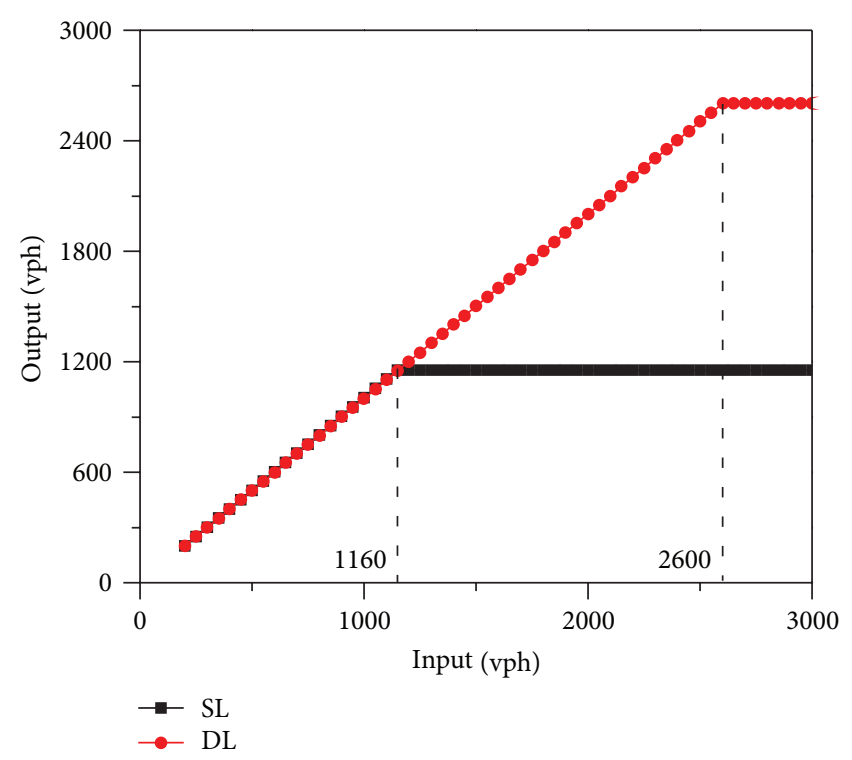

(b)

FIGURE 5: (a) Output of SL and DL model; (b) the fitting line of SL and DL model.

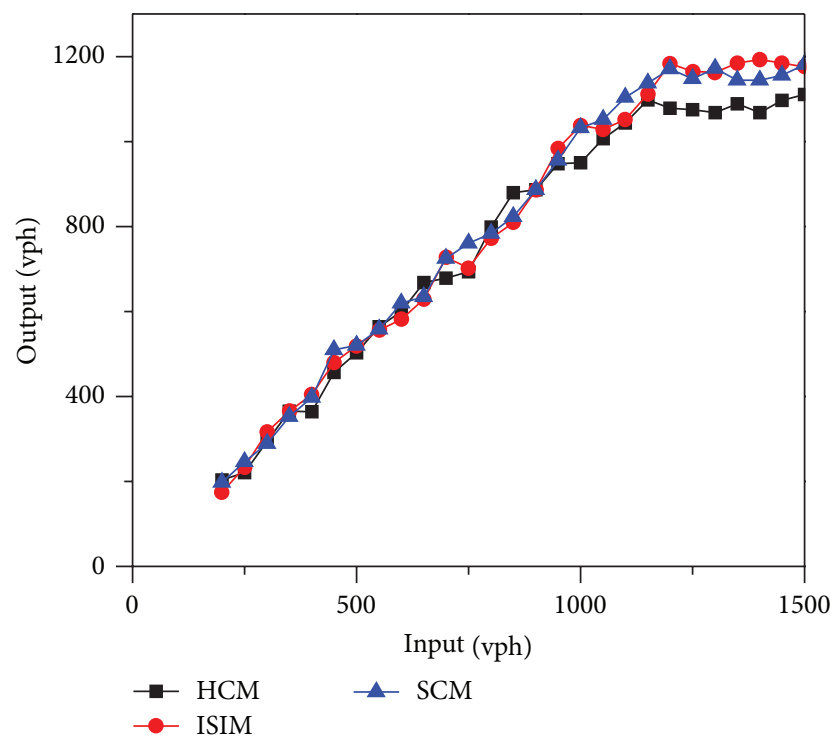

(a)

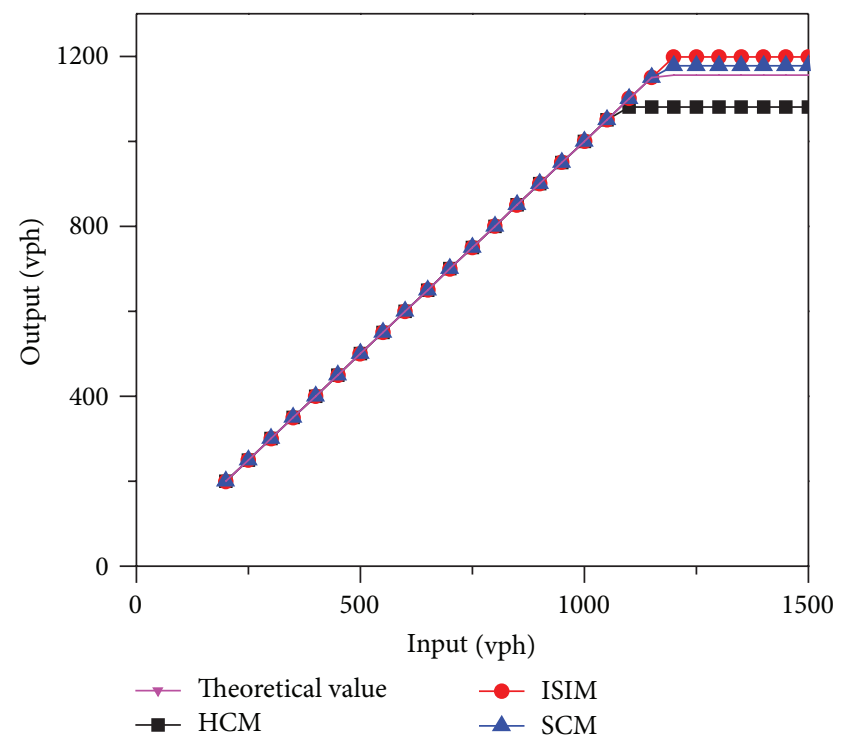

(b)

FIGURE 6: (a) Output results of three models; (b) fitting lines of three models and the theoretical value $\left(\right.$ Output $_{\text {max,ISIM }}=1200$ vph, Output $_{\max , \mathrm{SCM}}=1180 \mathrm{vph}$, Output ${ }_{\max , \mathrm{HCM}}=1080 \mathrm{vph}$, Output ${ }_{\text {max, theoretical }}=1160 \mathrm{vph}$ ).

also than the theoretical value. In HCM, vehicles have to slow down or even stop to wait the "PASS" signal, and finally this behavior results in the lowest output.

3.3. Output under Asymmetric Input Conditions. Next, we run three models under asymmetric input conditions as (13) and set the input as $1500 \mathrm{vph}$ according to the theoretical value mentioned above:

$$
\text { Input }_{\text {Lane 1 }}+\text { Input }_{\text {Lane 2 }} \leq 1500 \text { vph. }
$$

The results are presented as in Figure 7.

From Figure 7(b) we can find that HCM is sensitive to the asymmetric input. Once the inputs of two lanes are asymmetric, the output will decrease quickly. Then, we

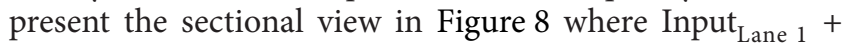
Input $_{\text {Lane 2 }}=1500 \mathrm{vph}$ for the better observation.

It is obvious from the Figure 8 that, for HCM, the output approaches the maximum when the inputs of each lanes are similarly equal, and it is noticed that the asymmetric input condition results in the huge fluctuation of the output. The 


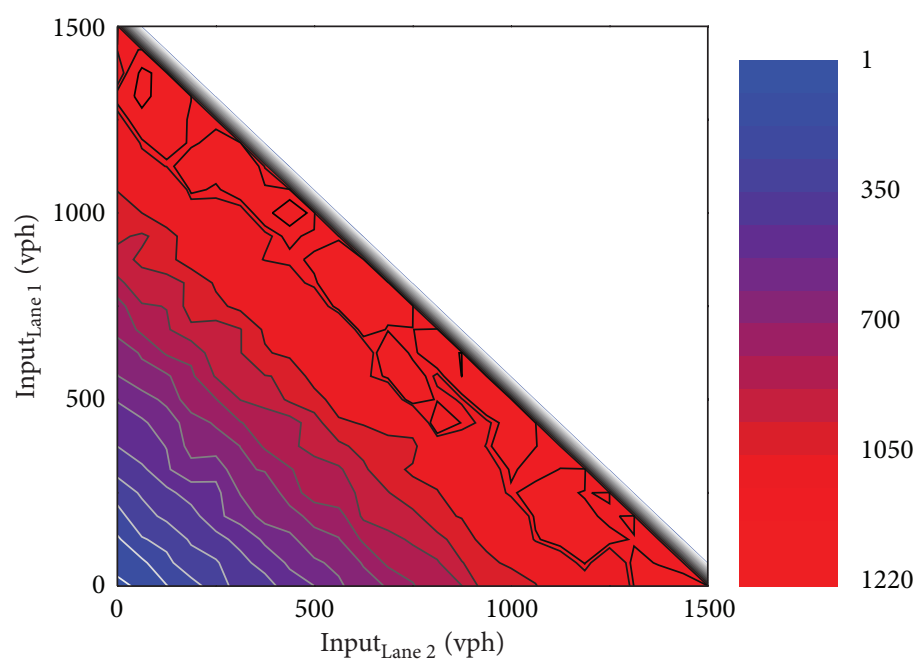

(a)

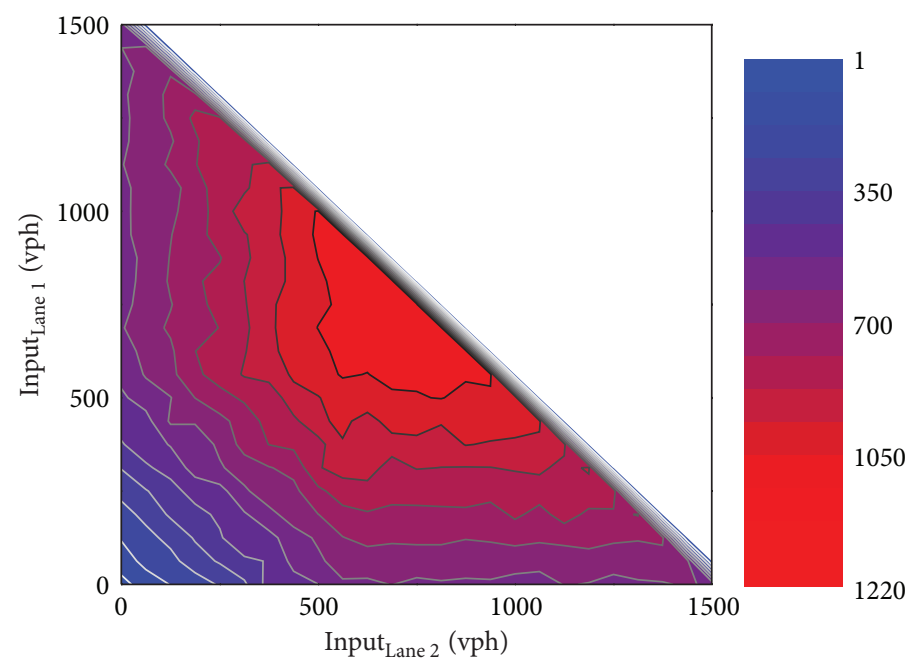

(b)

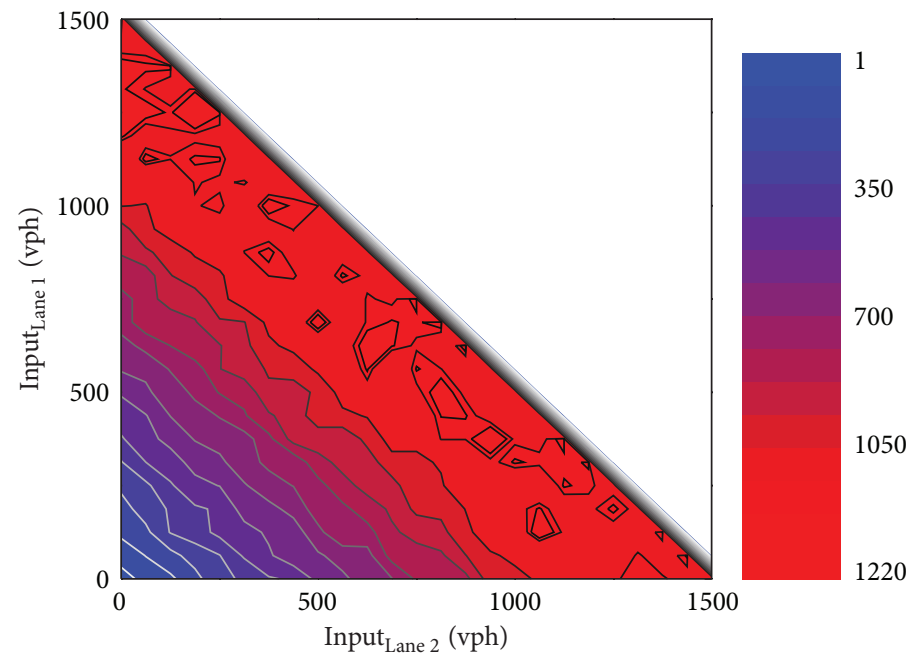

(c)

FIGURE 7: Output results of three models under asymmetric input conditions (a) ISIM, (b) HCM, and (c) SCM. 


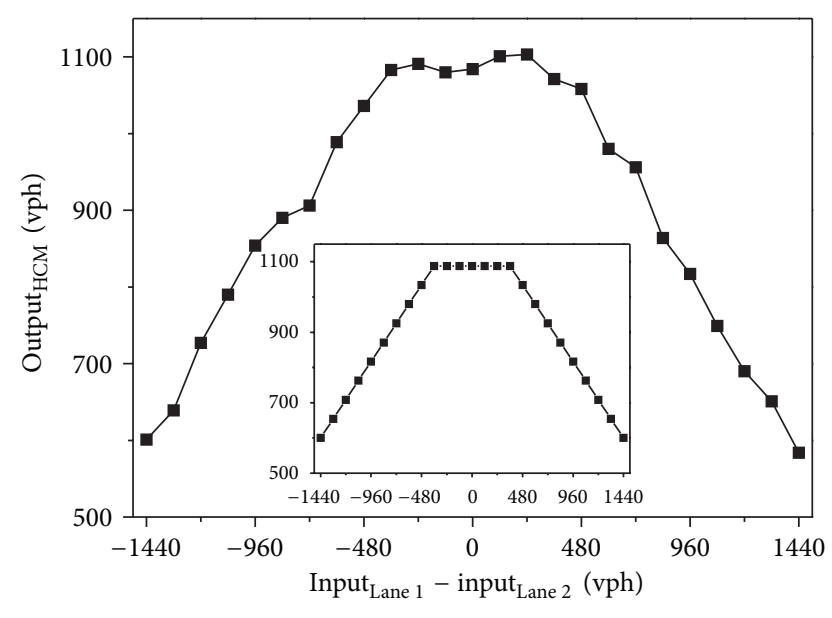

FIgURE 8: Output of HCM in the section where Input $_{\text {Lane } 1}+$ Input $_{\text {Lane 2 }}=1500 \mathrm{vph}$, and the insert shows its fitting line.

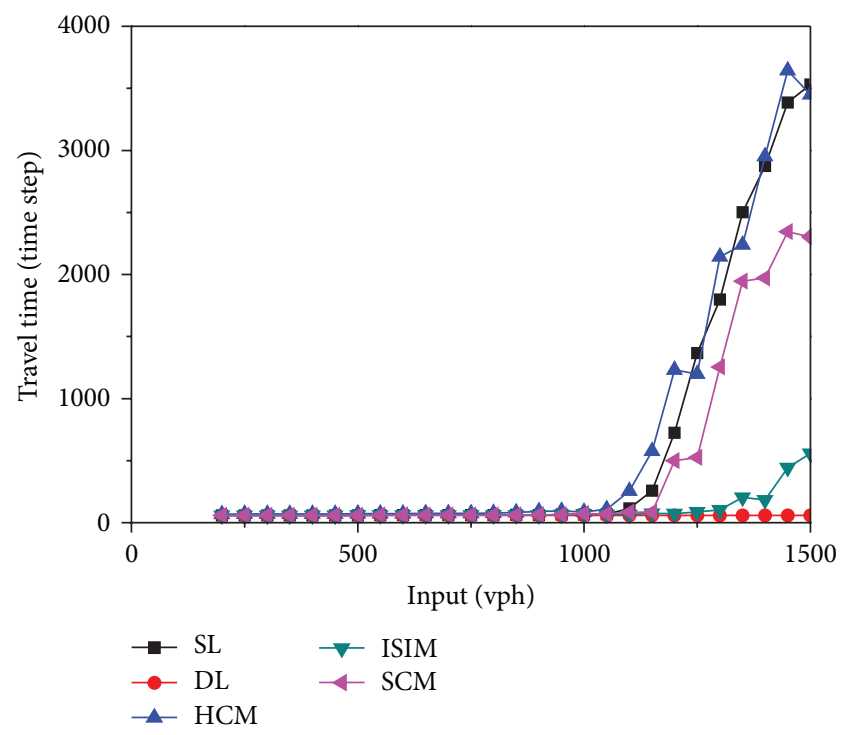

FIGURE 9: The travel time results of SL model, DL model, and three models.

maximum is bigger than the minimum by $27.3 \%$, and the descending slope measured from the fitting line in the insert is equal to -1 approximately. According to this, we should balance the input of each lane carefully when HCM model is implemented. SCM is not sensitive to the asymmetric input as the illustration. But we still can find out that when Input $_{\text {Lane 1 }}>$ Input $_{\text {Lane 2 }}$ the ouput of SCM is a bit better than that in the case of Input Lane $1<$ Input $_{\text {Lane } 2}$. It is the result that the vehicle has to change lane early when more vehicles are injected into Lane 1, and this behavior makes the DL model start to take effect passively. Compared with the previous two models, ISIM is more satisfactory. It is not sensitive to the asymmetric input condition and can get a better output result even than SCM. With the more optional lane-changing rules, vehicles on each lane can balance themselves spontaneously.

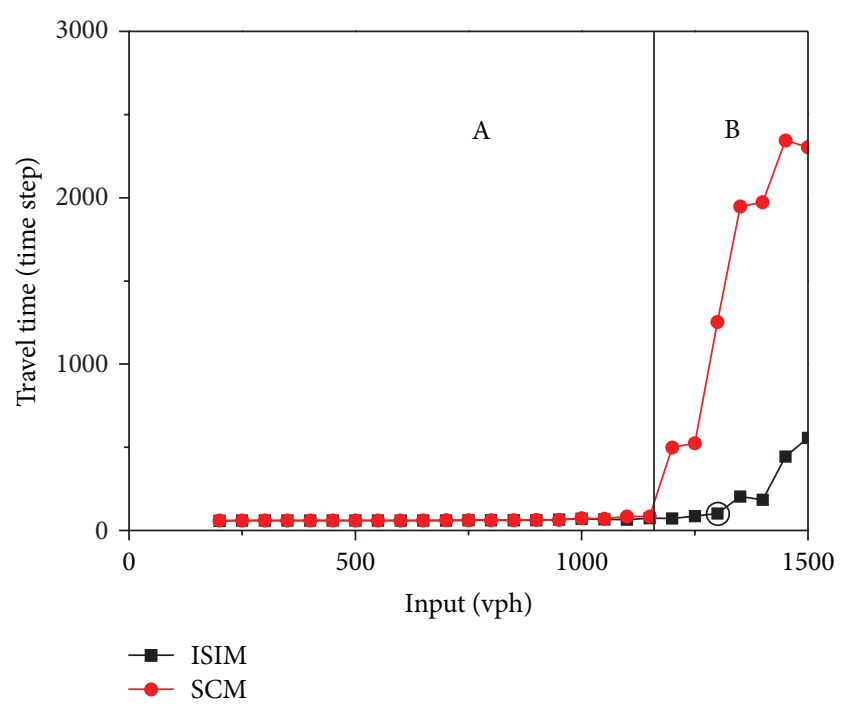

FIgURE 10: The travel time results of ISIM and SCM.

Thus the DL model works better, and the advantage will be more apparent when we focus on the travel time result.

3.4. Travel Time under Symmetric Input Conditions. Similar to the discussion of output, we simulate SL and DL model to obtain the theoretical travel time and the results of SL and DL model and other three models are presented in Figure 9.

When the input increases to their respective Output ${ }_{\max }$, the travel time begins to increase as shown in Figure 9 and the travel time of HCM increases especially quickly. It can be estimated that if the work zone model is well established, its travel time will be somewhere between the DL and SL model. Then we focus on the travel time results of three models. The results of HCM and SL model are overlapping, while the results of ISIM and SCM are optimized, and they both appear between the value of DL and SL model. According to this, we illustrate the results of ISIM and SCM in Figure 10 separately.

In Figure 10, we divide the input space into two areas according to the theoretical value of SL model:

$$
\begin{aligned}
& \text { Area A: } 0 \mathrm{vph}<\text { Input < } 1160 \mathrm{vph} \text {; } \\
& \text { Area B: } 1160 \mathrm{vph}<\text { Input < } 1500 \mathrm{vph} .
\end{aligned}
$$

In Area $\mathrm{A}$, the input is smaller than the theoretical value, and the travel time results of both models are about 58 time steps, so the mean velocity at which the vehicle passes through the system is about 3.4 lattice per time step. According to the maximum velocity (4 lattice per time step), it is a satisfactory value. In Area B, once the input is larger than the theoretical value, the travel time result of SCM increases dramatically. The result of ISIM is obviously better. The circled point (after which the result increases rapidly) of ISIM is about $1350 \mathrm{vph}$ which is about $200 \mathrm{vph}$ higher than the theoretical value and that of SCM. Furthermore, the travel time of ISIM is 2000 time steps less when the input reaches to $1500 \mathrm{vph}$, and this is a huge advantage to move vehicles in less time. It is noticed that the individual subjective initiative in ISIM can improve the velocity and capacity of the traffic. 


\section{Conclusions and Further Studies}

In this paper, we try to make a comparison between the three models: ISIM, HCM, and SCM. HCM model performs unsatisfactorily in both output and average travel time, because its control method is purely mechanical. The vehicle in it has to slow down or even stop for the signal, and the individual subjective initiative is not considered. When HCM is implemented, many factors should be taken into consideration, such as the careful input control, and the interval between the signal changes. Meanwhile, SCM model, owing to the passive performance of DL model, performs better than HCM model when the traffic is heavy. ISIM model performs the best among the three models under different traffic conditions due to the well-designed individual subjective initiative. The huge advantage in travel time results compared with SCM cannot be ignored. Vehicles in it can balance themselves more actively, and this makes the system perform the best in both output results, and travel time results. The individual subjective initiative is helpful for the vehicle to make a good use of system resources and make the system more flexible.

In further studies, we will try to take more details of ISIM model into consideration, such as the complex driver-vehicle behavior [17]. We try to establish a more realistic model and make a reasonable reference model for further studies.

\section{Acknowledgments}

This work is partially supported by the National Natural Science Foundation of China (70631002, 10774112) and the Program for New Century Excellent Talents in University (NCET-08-0406).

\section{References}

[1] A. Tarko, S. Kanipakapatman, and J. Wasson, "Modeling and optimization of the Indiana Lane merge control system on approaches to freeway work zones," Final Report FHWA/IN/ JTRP-97/12, Purdue University, West Lafayette, Ind, USA, 1998.

[2] P. T. McCoy, G. Pesti, and P. S. Byrd, "Alternative information to alleviate work zone related delays," SPR-PL-1 SPR-PL-1(35) P513, University of Nebraska-Lincoln, 1999.

[3] G. Pesti, D. R. Jessen, P. S. Byrd, and P. T. McCoy, "Traffic flow characteristics of the late merge work zone control strategy," Transportation Research Record, no. 1657, pp. 1-9, 1999.

[4] C. H. Walters, V. J. Pezoldt, K. N. Womack, S. A. Cooner, and B. T. Kuhn, "Understanding road rage: summary of first-year project activities," Tech. Rep. TX-01/4945-1, Texas Transportation Institute, 2000.

[5] A. Tarko and S. Venugopal, "Safety and capacity evaluation of the Indiana lane merge system," FHWA/iN/JTRP/-2000/19, Purdue University, West Lafayette, Ind, USA, 2001.

[6] T. Datta, K. Schattler, P. Kar, and A. Guha, "Development and Evaluation of an advanced dynamic lane merge traffic control system for 3 to 2 lane transition areas in work zones," Report RC-1451, Michigan Department of Transportation, 2004.

[7] A. G. Beacher, M. D. Fontaine, and N. J. Garber, "Field evaluation of late merge traffic control in work zones," Transportation Research Record, no. 1911, pp. 33-41, 2005.
[8] A. G. Beacher, M. D. Fontaine, and N. J. Garber, "Guidelines for using late merge traffic control in work zones: results of a simulation-based study," Transportation Research Record, no. 1911, pp. 42-50, 2005.

[9] S. Ishaka, Y. Qi, and P. Rayaproluc, "Safety evaluation of joint and conventional lane merge configurations for freeway work zones," Traffic Injury Prevention, vol. 13, no. 2, pp. 199-208, 2012.

[10] N. Yang, G. L. Chang, and K. P. Kang, "Simulation-based study on a lane-based signal system for merge control at freeway work zones," Journal of Transportation Engineering, vol. 135, no. 1, pp. 9-17, 2009.

[11] K. P. Kang and G. L. Chang, "Lane-based dynamic merge control strategy based on optimal thresholds for highway work zone operations," Journal of Transportation Engineering, vol. 135, no. 6, pp. 359-370, 2009.

[12] S. Wolfram, Theory and Applications of Cellular Automata, World Scientific, Singapore, 1986.

[13] S. Wolfram, A New Kind of Science, Wolfram Media, Champaign, Ill, USA, 2002.

[14] K. Nagel and M. Schreckenberg, "A cellular automaton model for freeway traffic," Journal de Physique, vol. 2, no. 12, pp. 22212229, 1992.

[15] N. Duffield, C. Lund, and M. Thorup, "Estimating flow distributions from sampled flow statistics," IEEE/ACM Transactions on Networking, vol. 13, no. 5, pp. 933-946, 2005.

[16] W. H. Wang, W. Zhang, H. W. Guo, H. Bubb, and K. Ikeuchi, "A safety-based behavioural approaching model with various driving characteristics," Transportation Research C, vol. 19, no. 6, pp. 1202-1214, 2011.

[17] W. Wang, F. Hou, H. Tan, and H. Bubb, "A framework for function allocations in intelligent driver interface design for comfort and safety," International Journal of Computational Intelligence Systems, vol. 3, no. 5, pp. 531-541, 2010. 


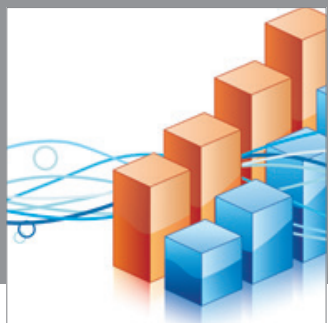

Advances in

Operations Research

mansans

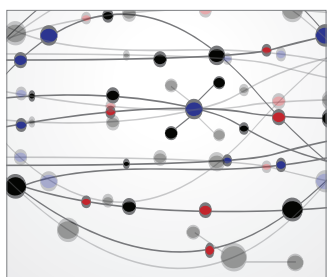

The Scientific World Journal
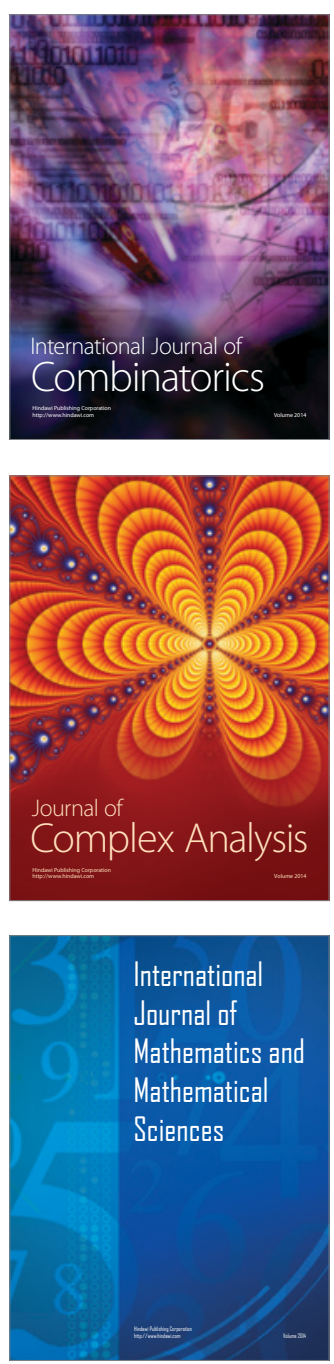
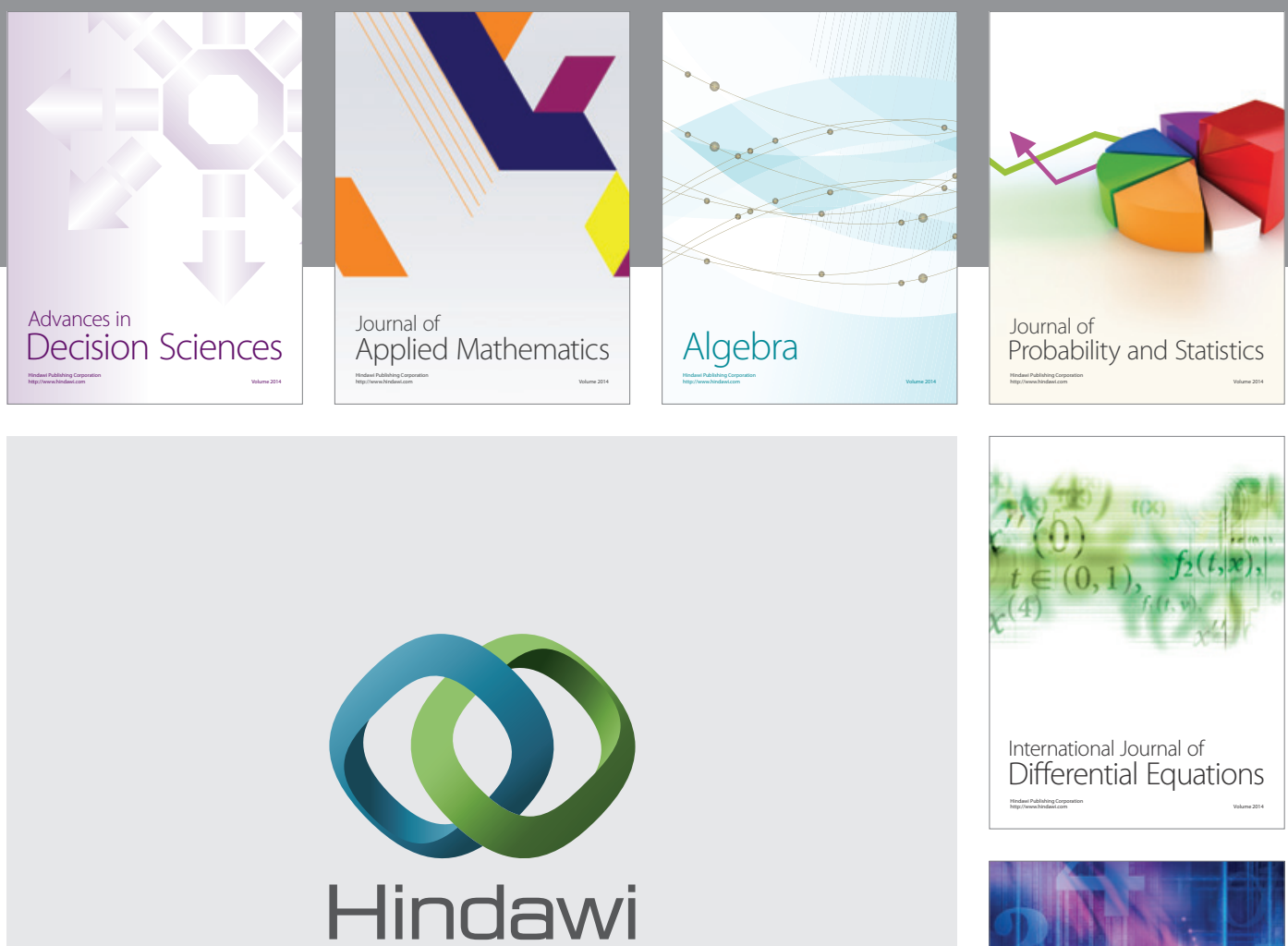

Submit your manuscripts at http://www.hindawi.com
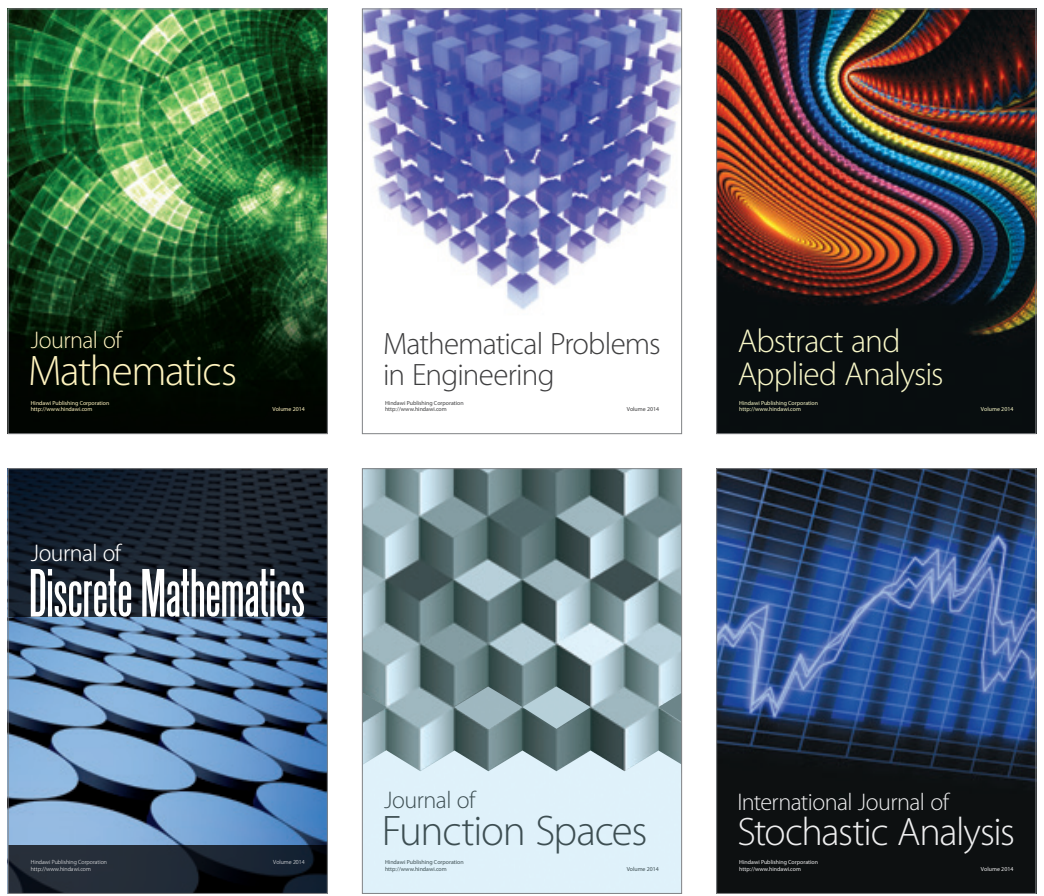

Journal of

Function Spaces

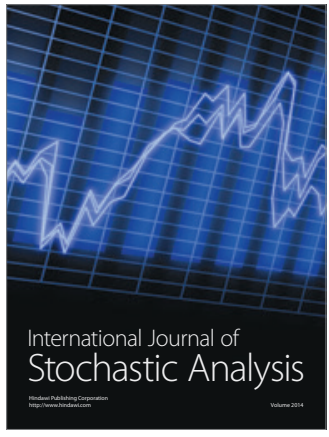

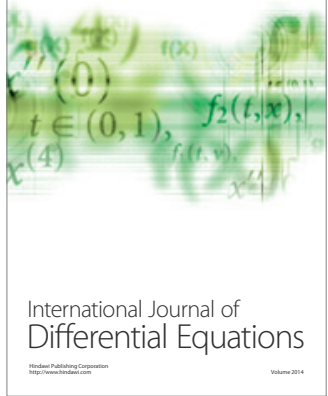
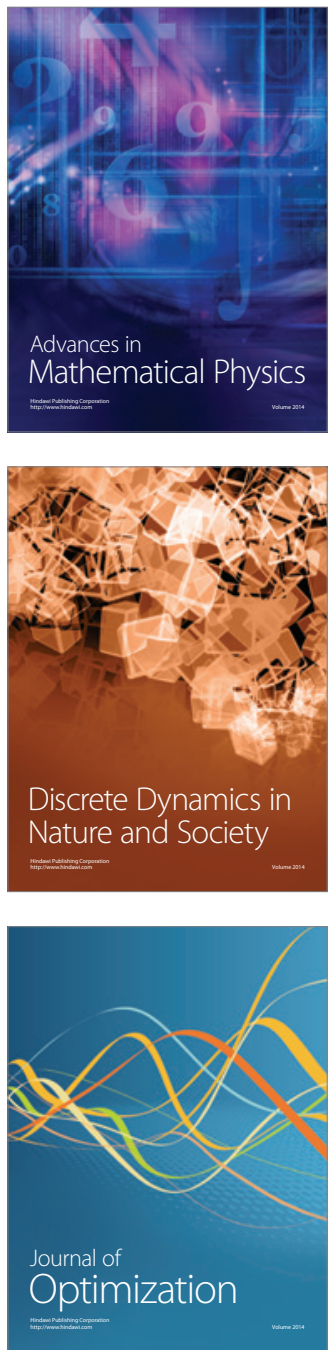